\title{
STEM characterization of Gold-Copper anisotropic nanocrystals
}

Lourdes Bazán-Díaz ${ }^{1,2}$, Rubén Mendoza-Cruz ${ }^{1,2}$, J.Jesús Velázquez-Salazar ${ }^{1}$, Ulises Santiago ${ }^{1}$, Daniel Bahena Uribe ${ }^{3}$, Miguel José-Yacamán ${ }^{1}$.

1. Department of Physics \& Astronomy, The University of Texas at San Antonio, One UTSA Circle, San Antonio, TX 78249, USA.

2. Instituto de Física, Universidad Nacional Autónoma de México, A. P. 20-364 Distrito Federal

C.P. 01000 México

3. Cinvestav. Av. Instituto Politécnico Nacional 2508, Col. San Pedro Zacatenco, Delegación Gustavo A. Madero, México D.F. Código Postal 07360

Alloy nanoparticles are an important group of nanomaterials exhibiting size, shape, structure and composition dependent properties. In bulk, gold-copper alloys exhibits ordered phases $\mathrm{Au}_{3} \mathrm{Cu}\left(\mathrm{L1}_{2}\right), \mathrm{AuCu}$ $\left(\mathrm{L1}_{0}\right), \mathrm{AuCu}_{3}\left(\mathrm{~L}_{2}\right)$ [1]. Theses phases could be modified in temperature and composition at the nanoscale. For instance, ligands play an important role in the synthesis of bi-metallic nanoparticles because they influence the final shape and size of the nanoparticle. The most common method used to synthesize alloyed nanoparticles is wet-chemistry that make use of phosphoric acids, polymeric chains or thiol groups as surfactants in organic solvents such as toluene that control the spatial and shape distribution of the nanoparticles. However, the knowledge of the internal structure of the crystals provides insights to understand the crystal growth of the nanosystem. In recent years it has been reported the relationship between shape and internal features such as stacking faults and twin boundaries on nanowires and decahedral particles [2]. This features (twins, stacking faults and other defects) can modified the final shape of nanoparticles. Some other factors that affect in the same way are the concentration of the reactants and the temperature ranges. Therefore, in this work we present a systematic study on gold-copper bimetallic system to analyze the internal structure of ultrathin nanowires, decahedral nanostars and nanocubes. Modifying few conditions during the synthesis, such as metal concentrations and surfactant used, namely hexadecylamine (HDA), octadecylamine (ODA), oleylamine (OLA) or 1-dodecanethiol (DDT), we obtained $\mathrm{Au}-\mathrm{Cu}$ nanocrystals with the different morphologies aforementioned. Through high resolution transmission electron microscopy (HRTEM), High Angle Annular Dark Field (HAADF) imaging and Energy Dispersive X-Ray Spectroscopy (EDS) we have obtained atomic resolution that allow us to describe the internal structure of the synthesized particles.

Fig. 1 shows a coiled ultrathin Au-Cu nanowire with diameter below $3 \mathrm{~nm}$ synthesized at low temperature. There have been few reports about similar nanostructures synthesized at high temperature [3-5]. However, to our knowledge, no previous reports have been done about this system so it represent by itself a novel and exceptionally interesting structure, since multiple twins and stacking faults are present along the whole wire and determine its growth.

Images in Fig. 2 correspond to pentagonal nanostars synthesized by using HDA (Fig. 2a) or OA (Fig.2b) and gold-copper nanocubes (Fig. 2c). From the high resolution images it was inferred that, in the case of the nanostars, the center of the particle consist of 5-fold-symmetry decahedral seeds and each branch grows along the twinning plane as is shown. Furthermore, the edge of each branch is reconstructed by stepped planes, which give rise to $\{433\}$ high index facets. On the other hand, when OLA is used, we obtain novel pentagonal nanoparticles similar to those made with HDA but the nanocrystals are smaller and the branches are rounded (Fig.2b). They also exhibit a core with 5-fold symmetry, but unlike the first 
ones, the branches grow along the [001] direction instead of the twinning plane of the 5-fold core. The branches show no twinning and its edges are formed by $\{111\}$ and $\{110\}$ stepped planes. In turn, goldcopper nanocubes were synthesized by using 1-dodecanethiol. The mean diameter of these cubes is around $8 \mathrm{~nm}$. They present flat $\{200\}$ flat faces and rest on the [001] axis zone as is shown in Fig. 2c.

In conclusion, we are able to obtain novel gold-copper nanocrystals with different morphologies by modifying the synthesis conditions and the surfactant employed. Undoubtedly, the knowledge of the structural arrangement at nanoscale level as well as the distribution of the elemental composition will bring a new way to understand the atomistic structures when bimetallic alloys are grown with twin boundaries and stacking faults.

\section{References:}

[1] Taherkhani F et al., Alloy Compd 617 (2014), p. 746.

[2] Ying C et al., Cryst. Growth Des 11 (2011),p.5457.

[3] Teng X, et al., Angew Chem Int Ed 47 (2008), p. 2055

[4] Velazquez-Salazar, J. J et al., ACS nano 5 (2011), p. 6272.

[5] Hong, X et al., Chem Commun 47 (2011), p. 5160.

[6] This project was supported by a grant from the National Institute on Minority Health and Health Disparities (G12MD007591) from the National Institutes of Health.
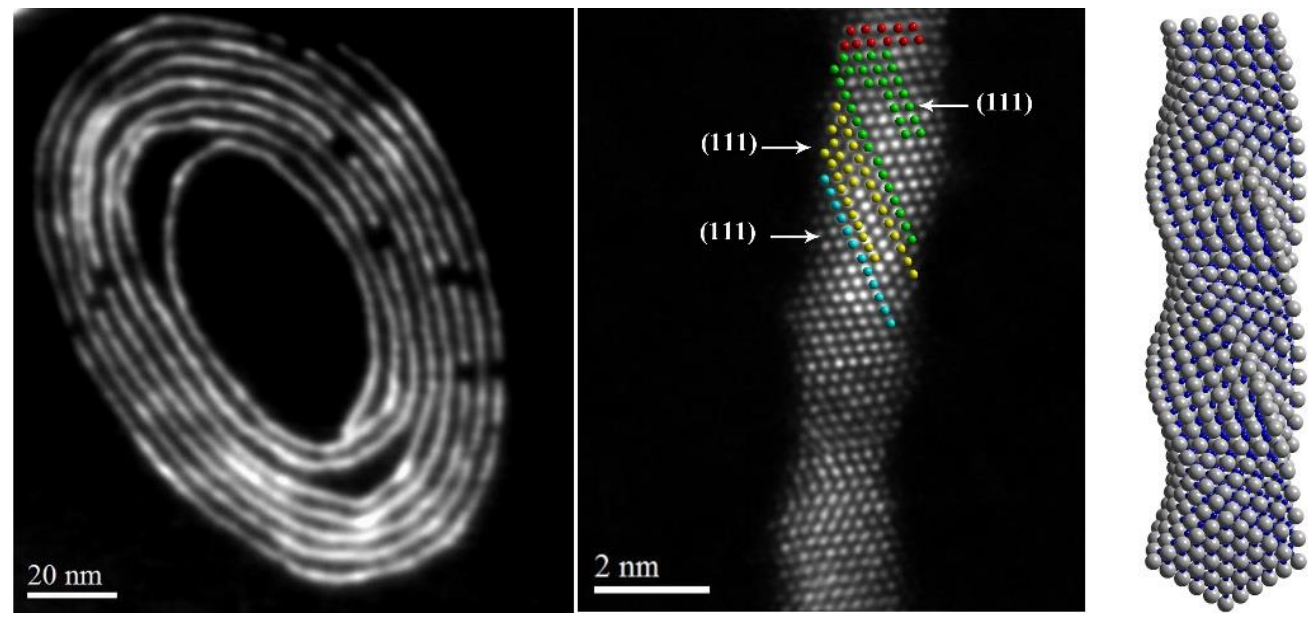

Fig. 1. Ultrathin gold-copper nanowire. Twinning and stacking faults rule its growth and final structure.
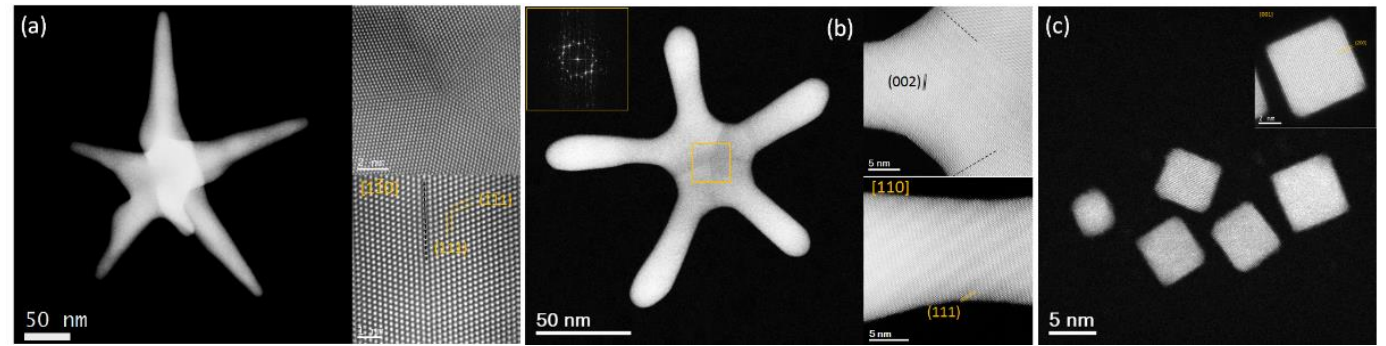

Fig. 2. Nanostars synthesized by using HDA (a) and OLA (b). It is noted the 5-fold symmetry of the core and the different direction of growth. (c) Nanocubes synthesized by using 1-dodecanethiol. 